practitioner for mental health related concerns. Satisfaction with follow-up care was dependant on participants' relationships with healthcare professionals and their perception of the continuity and comprehensiveness of care they received. Positive experiences of care included 'compassionate and supportive relationships', 'timely and comprehensive follow-up care' and 'inpatient care as a safe haven'. The establishment of trust in the services encouraged 'seeking help in crisis' and 'acceptance of and adherence to psychotropic medication'. Conversely, themes reflecting negative experiences of care included 'superficial and unsupportive relationships' and 'care lacking continuity and comprehensiveness' leaving some participants feeling isolated and unsupported. Unsupportive experiences within the services contributed to 'inhibited help-seeking' and 'reluctance or lack of adherence to psychotropic medication'. Participants with a history of self-harm and those with recent engagement with mental health services prior to the self-harm episode were more likely to report dissatisfaction with their interactions with healthcare professionals and with the level of care provided. Furthermore, those who described unsupportive or unsatisfactory care more frequently reported repeated selfharm, increased alcohol misuse and hopelessness for the future at follow-up.

Conclusion The study findings indicate that satisfaction with services, help-seeking and adherence to treatment may be improved by ensuring the consistent provision of timely, comprehensive and supportive aftercare following a high risk selfharm presentation. Absence of these aspects of care may contribute to ongoing distress and further suicidal behaviour.

\section{OP27 A NATIONAL CASE FATALITY STUDY OF DRUGS TAKEN IN INTENTIONAL OVERDOSE}

${ }^{1} \mathrm{CD}$ Daly*${ }^{*} \mathrm{EG}$ Griffin, ${ }^{1,2} \mathrm{PC}$ Corcoran, ${ }^{3,4} \mathrm{RW}$ Webb, ${ }^{3,5} \mathrm{DA}$ Ashcroft, ${ }^{2} \mathrm{PP}$ Perry, 1,2EA Arensman. 'National Suicide Research Foundation, National Suicide Research Foundation, Cork, Ireland; ${ }^{2}$ School of Public Health, University College Cork, Cork, Ireland; ${ }^{3}$ NIHR Greater Manchester Patient Safety Translational Research Centre, University of Manchester, Manchester, UK; ${ }^{4}$ Centre for Mental Health and Safety, University of Manchester, Manchester, UK; ${ }^{5}$ Centre for Pharmacoepidemiology and Drug Safety, University of Manchester, Manchester, UK

\subsection{6/jech-2019-SSMabstracts.27}

Background Intentional drug overdose (IDO) is the most common form of hospital-presenting self-harm and has been linked with marked increases in risk of dying by suicide and other causes. The type of drug taken in IDO is one of several factors that influence the likelihood of IDO repetition and fatality following overdose. Previous research examining the fatality of an overdose according to the drug types taken has attributed fatal toxicity to a number of psychotropic drugs. However, these findings have limited applicability as they focus on overdose acts involving single drugs, which represent a small minority of fatal IDOs. We aimed to describe the overdose characteristics of fatal and non-fatal IDO, and to establish which drug types are linked with greater risk of subsequent fatality.

Methods Data pertaining to 65,069 non-fatal IDO presentations from the National Self-Harm Registry, Ireland and 365 fatal IDOs from the National Drug-Related Deaths Index, for the period 1st Jan 2007 to 31st Dec 2014, were used to describe overdose characteristics of fatal and non-fatal IDOs, to calculate their incidence and to estimate case fatality risk ratios.
Results The risk of death following IDO was 1.7 times greater for males than females and fatal cases were on average nine years older than non-fatal cases, with each increasing year of age increasing the risk of a fatal outcome by $4.3 \%$. Multiple drug IDOs were over three times more likely to be fatal, compared to single drug IDOs. Tricyclic antidepressants were associated with a 15 -fold increased risk of death and opioids a 12-fold increased risk, relative to non-opioid analgesics (the reference category). Although the absolute risk of fatal outcome was higher for males than females, the elevation in risk was greater in females when tricyclic antidepressants or opioids were taken in IDO.

Conclusion Male gender, increasing age and multiple drug use were associated with fatal IDO outcome, and tricyclic antidepressants and opioids in particular were associated with a significantly increased risk of death following overdose. These findings inform the relative fatality risk of drugs that are commonly taken in intentional overdose, contributing to existing evidence-base in relation to safe and appropriate prescribing to patients who are at risk of self-harm. Together with the identification of the predictors of a fatal overdose outcome, these findings highlight areas for targeted intervention to prevent fatal overdose and also key areas for further research.

\section{OP28 ETHNICITY, SOCIODEMOGRAPHIC FACTORS, AND MENTAL HEALTH OUTCOMES IN YOUNG PEOPLE FROM THE MILLENNIUM COHORT STUDY}

G Ahmad*, J Das-Munshi. Psychological medicine, institute of psychiatry, psychology and neuroscience, king's college london, Iondon, uk

\subsection{6/jech-2019-SSMabstracts.28}

Background There is a socioeconomic gradient in mental health outcomes for young people: disadvantaged groups are more likely to suffer problems. In the UK, there is evidence of apparent resilience to mental health problems in young people from some ethnic minority groups, or lack of disadvantage despite facing greater socioeconomic adversity. This project investigated the relationship between social support, participation, and experiences of social adversity with mental health outcomes, focusing on inequalities by ethnicity, to explain any increased risk or resilience to adverse mental health outcomes.

Methods Data was analysed from 10,357 young people aged 14 taking part in the nationally representative Millennium Cohort Study. Univariable descriptive analysis assessed prevalence of mental health problems, identified using parent responses to the Strengths and Difficulties Questionnaire. Univariable logistic regression analysed crude associations of sociodemographic factors with mental health problems. Factors included: ethnicity, gender, socioeconomic status, parental relationship, time spent with friends, in organised activities, and religious attendance, self-reported social support, experience of victimisation, substance abuse, being a victim or perpetrator of bullying. Multivariable logistic regressions assessed if social factors explained any observed ethnic differences in mental health problems.

Results Social support and participation were associated with better mental health outcomes, and social adversity with worse mental health outcomes, for all cohort members at age 14 Overall, boys had increased odds for having mental health problems compared to girls (OR 1.24, 95\% CI 1.05-1.46). 
Adjusting for socioeconomic status revealed reduced ORs for mental health problems in the Pakistani group (girls 0.63, $0.41-0.99$; boys $0.49,0.27-0.89$ ), as well as Black African boys $(0.10,0.02-0.38)$, Indian boys $(0.40,0.21-0.77)$, and Bangladeshi girls $(0.18,0.05-0.65)$, compared to their White peers. After adjusting for social support, participation, and adversity factors, significantly reduced odds for mental health problems remained only for Black African boys (OR 0.16, 0.04-0.72).

Conclusion Socioeconomic status (SES) confounds resilience factors against mental health problems apparent in young people from some ethnic minority groups. Despite greater socioeconomic disadvantage, there was reduced prevalence of mental health problems for these young people after adjustment for SES. Furthermore, the changes to ORs after adjusting for social support, participation, and adversity factors suggest ethnic inequalities in mental health outcomes in this sample could be partly explained by these social factors. Further analysis is needed to investigate mediating mechanisms operating here. Social interventions may help foster resilience in young people against mental health problems, irrespective of ethnicity.

\section{OP29 INTERACTION BETWEEN SOCIOECONOMIC POSITION AND SOCIAL INTEGRATION IN SUICIDE MORTALITY: A NATIONALLY REPRESENTATIVE COHORT STUDY}

${ }^{1} \mathrm{C} \mathrm{Kim}{ }^{*},{ }^{2}$ J Dunn. ${ }^{1}$ Health Policy, McMaster University, Hamilton, Canada; ${ }^{2}$ Department of Health Aging and Society, McMaster University, Hamilton, Canada

10.1136/jech-2019-SSMabstracts.29

Background A low level of social integration and lower socioeconomic position (SEP) are well-known risk factors for suicidal behavior. Most literature has suggested that the effects of family types as a proxy of social integration and SEP measures on suicide are merely additive. However, as social integration is not dependent on SEP, the association of these effects could be interactive. Since the protective effects of social integration vary by gender, so could this interaction. The aim of this study was to examine the interaction between social integration, SEP and gender on suicide mortality in the Canadian context.

Methods Using data from the 1991 Canadian Census Health and Environment cohort (CanCHEC) —which included 2.5 million Canadians over a 20-year follow up period - we applied Cox proportional hazards regression models to examine the association among living arrangements (lives alone versus single parent family versus others), education (secondary versus non-secondary), income (low income versus non-low income), and employment status (unemployed versus else) by gender. Models were developed to observe how living arrangements attenuated the association between SEP measures and suicide. In the full model, we added interaction terms between living arrangements and employment status.

Results In model 1, which was only adjusted for age and three SEP measures, both men and women with low income (Hazard Ratio (HR): 1.846 [women], HR: 1.278 [Men]) and who were unemployed (HR: 1.501 [Women], HR: 1.677 [Men]) were more likely to be exposed to completed suicide. In all models, lower education was associated with suicide risk among men, but not among women. In model 2, when living arrangements were added, the association between SEP measures and suicide was much attenuated among women, but not among men. In the full model, an interactive effect between unemployment and living arrangements (living alone) was not shown among men. However, there was a significant interactive effect for women, demonstrating that unemployed women who do not live alone were 1.429 times more likely to complete suicide than employed women living with others, but women living alone and unemployed were 2.125 times more likely to do so.

Conclusion While SEP had more independent impacts from social integration on suicide among men, there were significant synergetic effects on suicide mortality among women in Canada.

\section{OP30 WHAT HAPPENS AFTER SELF-HARM? AN EXPLORATION OF SELF-HARM AND SUICIDE USING THE NORTHERN IRELAND REGISTRY OF SELF-HARM}

${ }^{1}$ A Maguire, ${ }^{1} \mathrm{~F}$ Tseliou, ${ }^{2} \mathrm{D}$ O'Hagan, ${ }^{1} \mathrm{D}$ O'Reilly, ${ }^{1} \mathrm{~S}$ McKenna*. ${ }^{1}$ Centre for Public Health, Queen's University, Belfast, UK; ${ }^{2}$ Public Health Agency, UK

\subsection{6/jech-2019-SSMabstracts.30}

Rationale Suicide is a major public health concern and Northern Ireland (NI) has the highest rate of both self-harm and suicide in the UK and Ireland. In order to target prevention strategies effectively it is vital to understand who is most at risk. Those who present with self-harm offer a prime opportunity for intervention. The aim of this study is to examine the risk factors for completed suicide following presentation with self-harm.

Data The Northern Ireland Registry of Self-Harm (NIRSH) collects information on all self-harm and suicide ideation presentations to all Emergency Departments in NI. NIRSH data for the four years 2012-2015 was linked to centralised electronic data relating to primary care, social services and prescribed medication and mortality records.

Methods Initial analyses describing the profile of those who present with self-harm was followed by logistic regression to quantify the likelihood of mortality with adjustment for factors associated with mental ill health and suicide risk.

Results The cohort consisted of all 1,483,435 individuals born or resident in NI from 1st January 1970 until $31^{\text {st }}$ December 2015 (maximum age in 2015, 45 years). During the follow-up period, $11,371(0.8 \%)$ individuals presented with self-harm and $1,719(0.1 \%)$ died by suicide. Rates of self-harm were equivalent for males and females with highest rates observed in the 18-24 years age group, and more common in deprived than affluent areas $(\mathrm{OR}=3.34,95 \% \mathrm{CI} 3.12,3.57)$. Rates of self-harm was highest among those who were (or ever had been) in the care of social services (OR $=12.06,95 \% \mathrm{CI} 11.26$, 12.93). Most individuals self-harm via self-poisoning with psychotropic medications (70.9\%), followed by self-injury with a sharp object $(21.6 \%)$. Although only $142(1.3 \%)$ of those who presented with self-harm went on to die by suicide, in the unadjusted model those who self-harmed were almost 12 times more likely to die by suicide compared to those who did not present with self-harm $(\mathrm{OR}=11.79$, 95\%CI 9.92,14.01). Suicide was more likely in individuals who selfharmed with more violent methods such as strangulation or drowning. After full adjustment for gender, age, social services care, area of residence and deprivation individuals who selfharm are still 7 times more likely to die by suicide $(\mathrm{OR}=7.05$, 95\%CI 5.88,8.45). 